\title{
Comparison Conventional Coated Beam with Functionally Graded Coated Beam
}

\author{
Abdolreza Toudehdehghan $^{1 *}$, Md. Mujibur Rahman ${ }^{2}$ \\ ${ }^{1}$ Department of Mechanical Engineering, Universiti Tenaga Nasional, km7, Jalan Kajang-Puchong, 43009 Kajang, Selangor, Malaysia \\ ${ }^{2}$ Faculty of Engineering and Quantity Surveying, INTI International University, Jalan BBN 12/1, Bandar Baru Nilai, 71800 Nilai, \\ Negeri Sembilan. Malaysia \\ *Corresponding author E-mail: abdolreza.toudehdehghan@newinti.edu.my
}

\begin{abstract}
New materials are essential for the development and advancement in material manufacturing technology. A brief overview of the history of human civilization shows that from stone tools to the steel age and then to the space age, had proven that the revolution of materials is key for new technology development. Today, it is known that phenomenon such as interface delamination and de-bonding on a conventional thermal barrier coating (which are present in an environment with high temperature) degrades the performance of the material and its mechanical properties. In overcoming this adverse effects, two or more types of materials such as ceramic and metal are composed together creating a type of composite named Functionally Graded Material (FGM) in the literature. In studying the behavior of FGM, models based on a theoretical derivation of Euler-Bernoulli beam theory using the superposition method clearly demonstrate the superiority of two different configurations of FGM against the conventional coated beam. The FGM coated and under coated models apply a power-law function on the material properties across the FGM layers in comparing the effects of thermo-mechanical loading to those of conventional coated beam. Specifically, the results show that FGM drastically reduces stress concentration preventing the initiation of any delamination or de-bonding.
\end{abstract}

Keywords: Euler-Bernoulli beam; FGM; Power-law function; Thermal barrier coating; Superposition method

\section{Introduction}

This document Functionally Graded Materials (FGMs) which are the composition of the component materials, usually gradually changing in one direction. Therefore, FGMs are considered as inhomogeneous composites that material properties differ continuously [1]. Due to change material properties with dimension in FGMs, by a function $\mathrm{f}(\mathrm{z})$ can be defined the material properties. This kind of materials were offered by a group of Japan scientists in 1984 [2], and extended by other scientists [3, 4]. In Figure one shows three kind of material functions in composite structures. The first graph, the function of material along the $\mathrm{z}$ direction is constant. It means the homogeneous materials (Figure 1.a). The second case, the composite materials are a combination of two different homogeneous materials (Figure 1.b). In the third one, the function of materials $\mathrm{f}(\mathrm{z})$ changes gradually along the $\mathrm{z}$ direction (Figure 1.c). Some researchers are considered FGM as a composition of several narrow layers [5]. FGMs are usually combining ceramics and metals which were at first considered as a thermal barrier for high-temperature surrounding. The composition of the ceramic/metal FGM, material properties from ceramic side gradually change to the metal side. This configuration of FGMs causes to decrease de-bonding between FGM layer and homogeneous layer of composite and also thermal stresses. The ceramic/metal FGM has ceramic characteristics like heat and corrosion resistance, and metal characteristics like high toughness and mechanical strength, simultaneously. Because of unique properties of ceramic/metal FGMs, The greatest community of researchers studied the thermal and mechanical properties of this kind of composites [6-9].
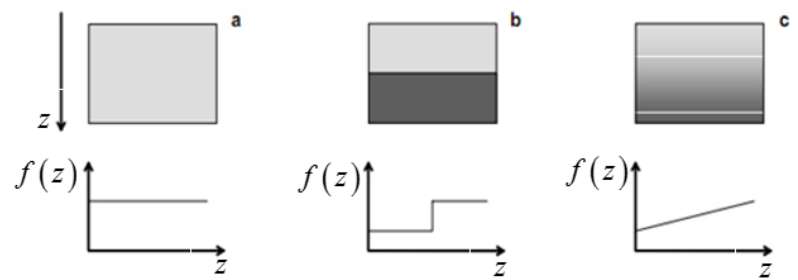

Fig. 1: Illustrative material function in three kind of structures: (a) homogenous material, (b) conventional composite (junction), (c) FGMs [10]

The intent of this article is to comparison and investigate the mechanical behavior of a clamped-clamped FGM coated rectangular beam and ceramic coated rectangular beam subjected to thermal transverse excitation and transverse mechanical loading (Figure 2).

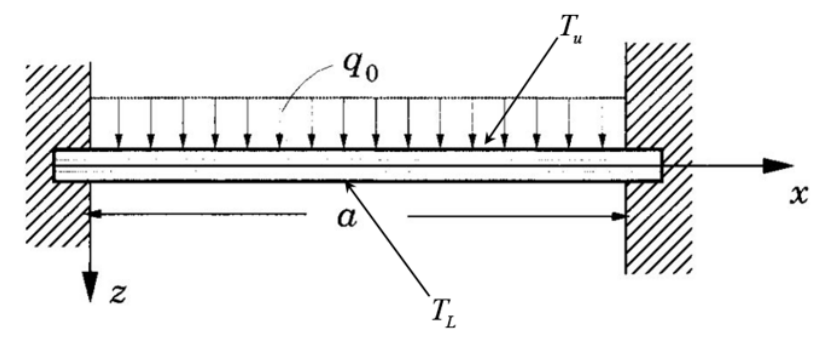

Fig. 2: Clamped coated beam under thermal and uniformed distributed load 
Material properties are function in the z-direction (thickness dimension). For FGM layer, the material properties are based on Power-law function. The ratio of the length of the beam to its thickness is fifty. Because of this ratio is more than 20, it will be considered as thin beam [11]. Therefore, Euler and Bernoulli Beam Theory (EBT) or Classical Beam Theory (CBT) is used to analyze the beam. This equation of FGM and the ceramic coated rectangular beam is solved by superposition method.

\section{Formulations}

\subsection{Material Properties of FGMs}

FGMs are mostly used as a thermal barrier in high-temperature surrounding and field. Under high- temperature environment, the material properties of the material will be changed proportion with up and down the temperature field. Thus, the material properties of FGMs are a function of temperature. These material properties such as thermal conductivity, the coefficient of thermal expansion, modulus of elasticity and etc., can be indicated as a nonlinear function of temperature $[12,13]$ :

$$
\begin{aligned}
& k(T)=P_{0}\left(P_{-1} T^{-1}+1+P_{1} T+P_{2} T^{2}+P_{3} T^{3}\right) \\
& \alpha(T)=P_{0}\left(P_{-1} T^{-1}+1+P_{1} T+P_{2} T^{2}+P_{3} T^{3}\right) \\
& E(T)=P_{0}\left(P_{-1} T^{-1}+1+P_{1} T+P_{2} T^{2}+P_{3} T^{3}\right)
\end{aligned}
$$

Where $P_{0}, P_{-1}, P_{1}, P_{2}$ and $P_{3}$ are temperature coefficients. The value of each coefficient are listed in table 1-3 for different ceram-

\begin{tabular}{|c|c|c|c|c|c|}
\hline Material & $\mathrm{P}_{0}$ & $\mathrm{p}_{-1}$ & $\mathrm{P}_{1}$ & $\mathrm{P}_{2}$ & $\mathrm{P}_{3}$ \\
\hline Zirconia & 1.7000 & 0 & $\underbrace{1.276 \times 10^{-}}_{4}$ & $6.648 \times 10^{-}$ & 0 \\
\hline $\begin{array}{l}\text { Aluminum } \\
\text { oxide }\end{array}$ & $\begin{array}{c}- \\
14.087\end{array}$ & -1123.6 & $\underset{3}{-227 \times 10^{-}}$ & 0 & 0 \\
\hline $\begin{array}{l}\text { Silicon } \\
\text { Nitride }\end{array}$ & 13.723 & 0 & $1.032 \times 10^{-}$ & $\underset{7}{5.466 \times 10^{-}}$ & $\begin{array}{c}- \\
7.876 \times 10^{-}\end{array}$ \\
\hline Ti-6Al-4V & 1.0000 & 0 & $\underset{2}{1.704 \times 10^{-}}$ & 0 & 0 \\
\hline $\begin{array}{c}\text { Stainless } \\
\text { Steel }\end{array}$ & 15.379 & 0 & $\frac{-}{-264 \times 10^{-}}$ & $\underset{6}{2.092 \times 10^{-}}$ & $\begin{array}{c}- \\
7.223 \times 10^{-}\end{array}$ \\
\hline Nickel $^{\text {a }}$ & 187.66 & 0 & $\underset{3}{-}{ }^{-}$ & $4.005 \times 10^{-}$ & $\begin{array}{c}- \\
1.983 \times 10^{-}\end{array}$ \\
\hline Nickel $^{b}$ & 58.754 & 0 & $\frac{-}{4.614 \times 10^{-}}$ & $\underset{7}{6.670 \times 10^{-}}$ & $\begin{array}{c}- \\
1.523 \times 10^{-}\end{array}$ \\
\hline
\end{tabular}
ics and metals [14].

Table 1: Thermal conductivity of ceramics and metals (W/m K)

\begin{tabular}{|c|c|c|c|c|c|}
\hline Material & $\mathrm{P}_{0}$ & $\mathrm{p}_{-1}$ & $\mathrm{P}_{1}$ & $\mathrm{P}_{2}$ & $\mathrm{P}_{3}$ \\
\hline Zirconia & $244.27 \times 10^{9}$ & 0 & $1.37 \underset{3}{-}$ & $1.214 \times 6$ & $\underset{10}{3.681 \times 10}$ \\
\hline $\begin{array}{c}\text { Aluminum } \\
\text { oxide }\end{array}$ & $349.55 \times 10^{9}$ & 0 & $3.853 \times 40^{-}$ & $\underset{7}{4.027 \times 10}$ & $\begin{array}{c}- \\
1.673 \times 10 \\
10\end{array}$ \\
\hline $\begin{array}{l}\text { Silicon } \\
\text { Nitride }\end{array}$ & $348.43 \times 10^{9}$ & 0 & $3.070 \times 10^{-}$ & $2.160 \times 10$ & $\underset{11}{8.946 \times 10}$ \\
\hline Ti-6Al-4V & $122.56 \times 10^{9}$ & 0 & $\frac{-}{4.586 \times 10^{-}}$ & 0 & 0 \\
\hline $\begin{array}{c}\text { Stainless } \\
\text { Steel }\end{array}$ & $201.04 \times 10^{9}$ & 0 & $3.079 \times 10^{-}$ & $\begin{array}{c}- \\
6.534 \times 10\end{array}$ & 0 \\
\hline
\end{tabular}

\begin{tabular}{|c|c|c|c|c|c|}
\hline Nickel & $223.95 \times 10^{9}$ & 0 & $\begin{array}{c}- \\
2.794 \times 10 \\
4\end{array}$ & $\underset{9}{3.998 \times 10}$ & 0 \\
\hline
\end{tabular}

Table 2: Coefficient of thermal expansion of ceramics and metals $(1 / \mathrm{K})$

\begin{tabular}{|c|c|c|c|c|c|}
\hline Material & $\mathrm{P}_{0}$ & $\mathrm{p}_{-1}$ & $\mathrm{P}_{1}$ & $\mathrm{P}_{2}$ & $\mathrm{P}_{3}$ \\
\hline Zirconia & $12.766 \times 10$ & 0 & $\frac{-}{1.491 \times 10^{-}}$ & $\underset{5}{1.006 \times 10^{-}}$ & $\begin{array}{c}- \\
6.778 \times 10 \\
11\end{array}$ \\
\hline $\begin{array}{l}\text { Aluminum } \\
\text { oxide }\end{array}$ & $\underset{6}{6.8269 \times 10}$ & 0 & $\underset{4}{1.838 \times 10^{-}}$ & 0 & 0 \\
\hline $\begin{array}{l}\text { Silicon } \\
\text { Nitride }\end{array}$ & $\underset{6}{5.8723 \times 10}$ & 0 & $\underset{4}{9.095 \times 10^{-}}$ & 0 & 0 \\
\hline Ti-6Al-4V & $7.5688 \times 10$ & 0 & $6.638 \times 10^{-}$ & $3.147 \times 6 \times 10^{-}$ & 0 \\
\hline $\begin{array}{c}\text { Stainless } \\
\text { Steel }\end{array}$ & $12.330 \times 10 \times 10$ & 0 & $\underset{4}{8.086 \times 10}$ & 0 & 0 \\
\hline Nickel & $\underset{6}{9.9209 \times 10}$ & 0 & $8.705 \times 10^{-}$ & 0 & 0 \\
\hline
\end{tabular}

Table 3: Modulus of elasticity of ceramics and metals $(\mathrm{Pa})$

FGM is a composition of metal and ceramic volume fraction which is represented in relation to

$V_{m}+V_{c}=1$

Power-law distribution is a mathematical function of the volume fraction of the composition. This function has been used broadly in several numbers of research investigation by several researchers [15-19]. The volume fraction of the power-law function of FGM structures can be written as below [20]:

$$
V_{c}=g(z)=\left(\frac{2 z+h}{2 h}\right)^{n}
$$

Where $\mathrm{h}$ is the thickness of the FGM structure and $\mathrm{n}$ is the material parameter. When $n=0$ the FGM layer is a fully metal layer or when $n=\infty$ the layer is fully ceramic. Thus based on the power law function the material properties vary depending on the position and amount of $n$ in the layer. Therefore, the material properties are varied through the thickness of FGM layer as follow according to power law function.

$$
\begin{aligned}
& E_{f}(z, T)=E_{c}(T)+\left(E_{m}(T)-E_{c}(T)\right)\left(\frac{2 z+h}{2 h}\right)^{n} \\
& \alpha_{f}(z, T)=\alpha_{c}(T)+\left(\alpha_{m}(T)-\alpha_{c}(T)\right)\left(\frac{2 z+h}{2 h}\right)^{n} \\
& k_{f}(z, T)=k_{c}(T)+\left(k_{m}(T)-k_{c}(T)\right)\left(\frac{2 z+h}{2 h}\right)^{n}
\end{aligned}
$$

Where $E_{c}, \alpha_{c}, k_{c}$ are the modulus of elasticity, thermal expansion, and thermal conductivity of ceramic as shown in Figure 3, respectively at the top of the FGM layer $\left(z=-\frac{h}{2}\right)$ and $E_{m}, \alpha_{m}, k_{m}$ are modulus elasticity, thermal expansion, and thermal conductivity of metal respectively at the bottom of the FGM layer $\left(z=\frac{h}{2}\right)$. It should be noted, Poisson's ratio is considered to be a constant for composite structure [21].

The distribution of material properties in the thickness direction of FGM layer with power-law function are plotted in Figure 4 to 6. 


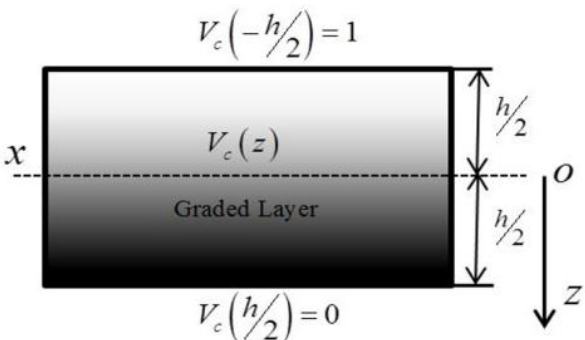

Fig. 3: Material properties throughout the thickness of FGM layer [22]

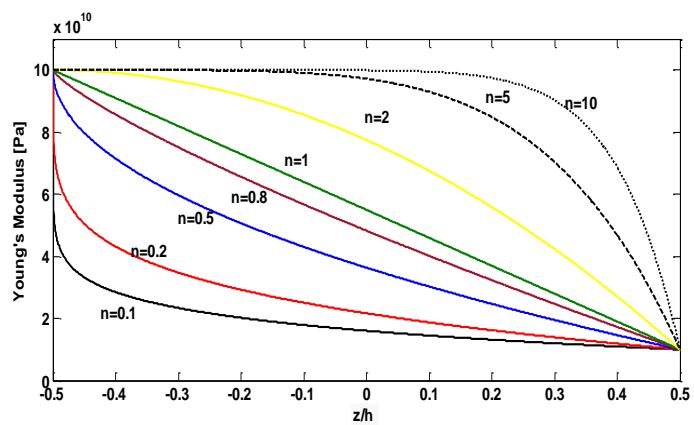

Fig. 4: The dispensation of Young's Modulus in the thickness direction of FGM layer

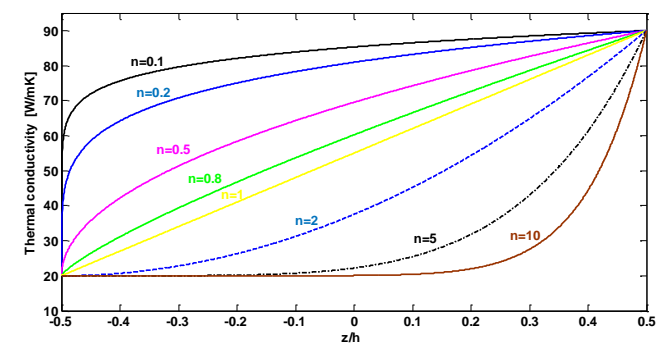

Fig. 5: The dispensation of thermal conductivity in the thickness direction of FGM layer

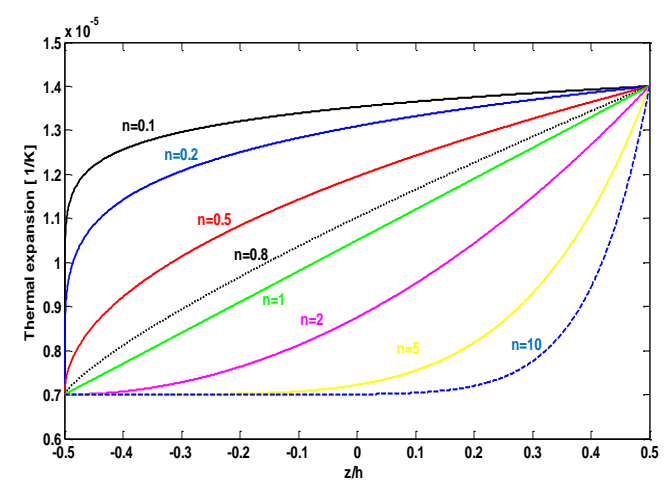

Fig. 6: The dispensation of Thermal expansion coefficient in the thickness direction of FGM layer

\subsection{Composite Models of Beam Structure}

Three composite models as a Thermal Barrier Coating (TBC) are considered in beam structure. These three models are based on the arrangement of the coated layer was categorized. These three models of the coating are as follows:

\subsubsection{Homogeneous Coating (HC) Model}

This model is consisting of substrate layer at the bottom and ceramic homogeneous coating layer at the top of the beam as shown in Figure 7. The diversification of material properties through the thickness is expounded by [21]:

$$
\begin{aligned}
& E(z, T)=E_{h c}(T) \quad \text { for } \quad-\frac{h_{1}+h_{3}}{2} \leq z \leq \frac{h_{3}-h_{1}}{2} \\
& E(z, T)=\frac{E_{h c}(T)+E_{h}(T)}{2} \quad \text { for } \quad z=\frac{h_{3}-h_{1}}{2} \\
& E(z, T)=E_{h}(T) \quad \text { for } \quad \frac{h_{3}-h_{1}}{2} \leq z \leq \frac{h_{1}+h_{3}}{2} \\
& \alpha(z, T)=\alpha_{h c}(T) \quad \text { for } \quad-\frac{h_{1}+h_{3}}{2} \leq z \leq \frac{h_{3}-h_{1}}{2} \\
& \alpha(z, T)=\frac{\alpha_{h c}(T)+\alpha_{h}(T)}{2} \quad \text { for } \quad z=\frac{h_{3}-h_{1}}{2} \\
& \alpha(z, T)=\alpha_{h}(T) \quad \text { for } \quad \frac{h_{3}-h_{1}}{2} \leq z \leq \frac{h_{1}+h_{3}}{2}
\end{aligned}
$$

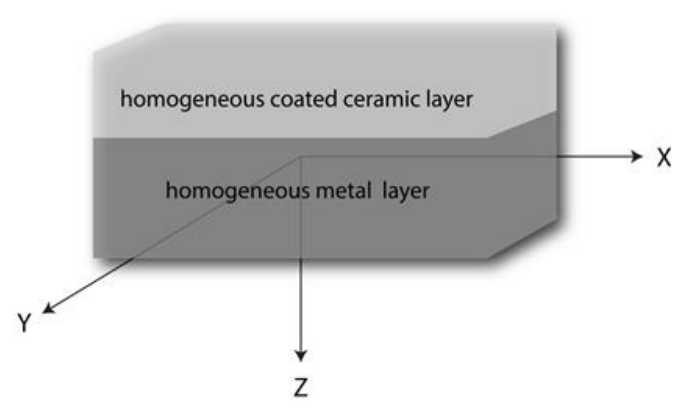

Fig. 7: Isometric sketch of $\mathrm{HC}$ model [23]

$$
\begin{aligned}
& k(z, T)=k_{h c}(T) \quad \text { for } \quad-\frac{h_{1}+h_{3}}{2} \leq z \leq \frac{h_{3}-h_{1}}{2} \\
& k(z, T)=\frac{k_{h c}(T)+k_{h}(T)}{2} \quad \text { for } \quad z=\frac{h_{3}-h_{1}}{2} \\
& k(z, T)=k_{h}(T) \quad \text { for } \quad \frac{h_{3}-h_{1}}{2} \leq z \leq \frac{h_{1}+h_{3}}{2}
\end{aligned}
$$

\subsubsection{Functionally Graded Coating (FGC) Model}

In FGC.model, FGM layer is located as a coated layer on top and homogeneous substrate layer on the bottom of the beam (Figure 8). The variation of material properties through the thickness is expound by:

$$
\begin{gathered}
E_{f}(z, T)=E_{h c}(T)+\left(E_{h}(T)-E_{h c}(T)\right)\left(\frac{z-\left(-\frac{h_{1}+h_{2}}{2}\right)}{h_{2}}\right)^{n} \\
\text { for } \quad-\frac{h_{1}+h_{2}}{2} \leq z \leq \frac{h_{2}-h_{1}}{2} \\
E(z, T)=E_{h}(T) \quad \text { for } \quad \frac{h_{2}-h_{1}}{2} \leq z \leq \frac{h_{1}+h_{2}}{2}
\end{gathered}
$$

$\alpha_{f}(z, T)=\alpha_{h c}(T)+\left(\alpha_{h}(T)-\alpha_{h c}(T)\right)\left(\frac{z-\left(-\frac{h_{1}+h_{2}}{2}\right)}{h_{2}}\right)^{\prime \prime}$

$$
\text { for } \quad-\frac{h_{1}+h_{2}}{2} \leq z \leq \frac{h_{2}-h_{1}}{2}
$$

$$
\alpha(z, T)=\alpha_{h}(T) \quad \text { for } \quad \frac{h_{2}-h_{1}}{2} \leq z \leq \frac{h_{1}+h_{2}}{2}
$$




$$
\begin{aligned}
& k_{f}(z, T)=k_{h c}(T)+\left(k_{h}(T)-k_{h c}(T)\right)\left(\frac{z-\left(-\frac{h_{1}+h_{2}}{2}\right)}{h_{2}}\right)^{n} \\
& \text { for } \quad-\frac{h_{1}+h_{2}}{2} \leq z \leq \frac{h_{2}-h_{1}}{2} \\
& k(z, T)=k_{h}(T) \quad \text { for } \quad \frac{h_{2}-h_{1}}{2} \leq z \leq \frac{h_{1}+h_{2}}{2}
\end{aligned}
$$

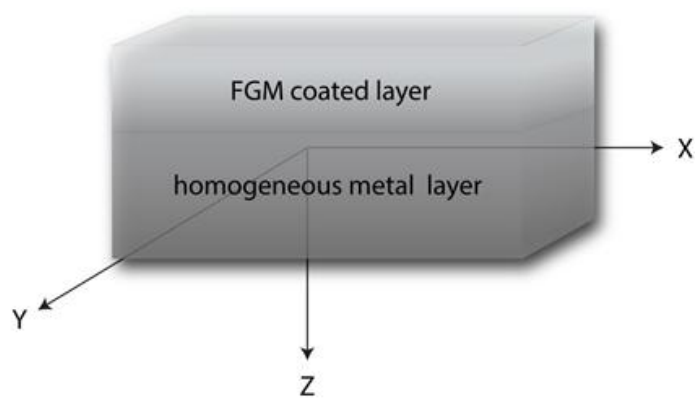

Fig. 8: Isometric sketch of FGC model [21]

\subsubsection{Homogeneous-Functionally Graded Coating (H-FGC) Model}

H-FGC model consists of three layers. The ceramic coated layer, FGC layer and substrate layer which are located from top to bottom of the beam respectively (Figure 9). The variety of material properties through the thickness is expounded by:

$$
\begin{aligned}
& E(z, T)=E_{h c}(T) \quad \text { for } \quad-\frac{h_{1}+h_{2}+h_{3}}{2} \leq z \leq \frac{h_{3}-h_{1}-h_{2}}{2} \\
& E_{f}(z, T)=E_{h c}(T)+\left(E_{h}(T)-E_{h c}(T)\right)\left(\frac{z-\left(\frac{h_{3}-h_{1}-h_{2}}{2}\right)}{h_{2}}\right)^{n} \\
& \text { for } \quad \frac{h_{3}-h_{1}-h_{2}}{2} \leq z \leq \frac{h_{2}+h_{3}-h_{1}}{2} \\
& E(z, T)=E_{h}(T) \quad \text { for } \quad \frac{h_{2}+h_{3}-h_{1}}{2} \leq z \leq \frac{h_{1}+h_{2}+h_{3}}{2} \\
& \alpha(z, T)=\alpha_{h c}(T) \quad \text { for } \quad-\frac{h_{1}+h_{2}+h_{3}}{2} \leq z \leq \frac{h_{3}-h_{1}-h_{2}}{2} \\
& \alpha_{f}(z, T)=\alpha_{h c}(T)+\left(\alpha_{h}(T)-\alpha_{h c}(T)\right)\left(\frac{z-\left(\frac{h_{3}-h_{1}-h_{2}}{2}\right)}{h_{2}}\right)^{n} \\
& \text { for } \quad \frac{h_{3}-h_{1}-h_{2}}{2} \leq z \leq \frac{h_{2}+h_{3}-h_{1}}{2} \\
& \alpha(z, T)=\alpha_{h}(T) \quad \text { for } \quad \frac{h_{2}+h_{3}-h_{1}}{2} \leq z \leq \frac{h_{1}+h_{2}+h_{3}}{2} \\
& k(z, T)=k_{h c}(T) \quad \text { for } \quad-\frac{h_{1}+h_{2}+h_{3}}{2} \leq z \leq \frac{h_{3}-h_{1}-h_{2}}{2} \\
& k_{f}(z, T)=k_{h c}(T)+\left(k_{h}(T)-k_{h c}(T)\right)\left(\frac{z-\left(\frac{h_{3}-h_{1}-h_{2}}{2}\right)}{h_{2}}\right)^{n} \\
& \text { for } \quad \frac{h_{3}-h_{1}-h_{2}}{2} \leq z \leq \frac{h_{2}+h_{3}-h_{1}}{2} \\
& k(z, T)=k_{h}(T) \quad \text { for } \quad \frac{h_{2}+h_{3}-h_{1}}{2} \leq z \leq \frac{h_{1}+h_{2}+h_{3}}{2}
\end{aligned}
$$

\subsection{Governing Equation of Beam}

The hypotheses of the EBT or CBT, similar to the Kirchhoff hypothesis that experimental observations present that these hypothesizes are reasonable for long, slender beams consist of isotropic 
materials with solid cross-sections. The kinematic assumption will be as $[25,26]$ :

- The deflection of the beam $w_{0}(x, z)$ (vertical displacement a point on the mid-plane) of any points on the cross-section are linear (Figure 10).

- After bending the deformation (lateral and longitudinal displacement) throughout the mid-plane of the beam is zero.

- The normal cross-sections to mid-plane of the beam after deformation will be remain plane and orthogonal to the beam $\mathrm{x}$-axis (Figure 10).

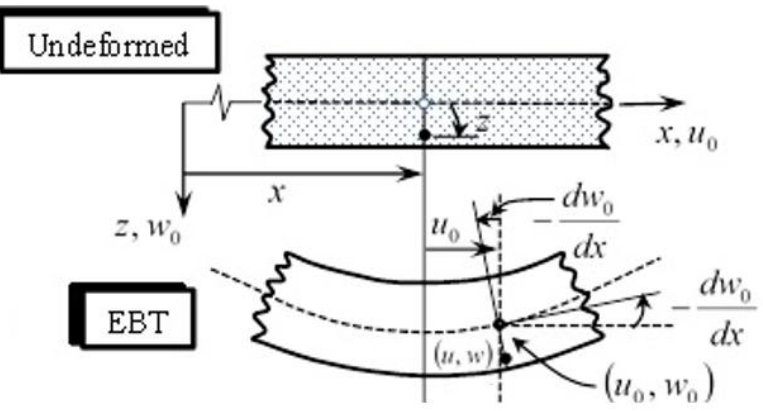

Fig. 10: Deformation of cross-section in EBT [26]]

At any point of the beam in the $\mathrm{z}$ and $\mathrm{x}$ directions displacements are

$$
\begin{aligned}
& u(x, y, z)=u_{0}(x, y)-z \frac{d w(x)}{d x} \\
& v(x, y, z)=0 \\
& w(x, y, z)=w_{0}(x)
\end{aligned}
$$

In Figure $10, u_{0}(x, y)$ is shown as a displacement on the midplane and because of the second assumption, it is equal to zero. As a consequence of the assumptions, shear strain $\varepsilon_{x y}$ and normal strain $\varepsilon_{z}$ are negligible. Thus the strain relations can be expressed as:

$$
\begin{aligned}
& \varepsilon_{x x}=\frac{d u}{d x}=-z \frac{d^{2} w}{d x^{2}}=-z \kappa_{x} \\
& \varepsilon_{y y}=\varepsilon_{x y}=\varepsilon_{z z}=0
\end{aligned}
$$

Where $\kappa_{x}$ is the curvature of the beam.

Based on Hooke's law, the stress-strain relation for FGM beam in plane stress condition is

$$
\sigma_{x x}=E(z, T)\left[-z \kappa_{x}-\alpha(z, T) T(z)\right]
$$

The concept of bending moment is defined as

$$
M_{x}=\int_{-\frac{h}{2}}^{\frac{h}{2}} b z \sigma_{x x} d z
$$

Where, $h$ and $b$ respectively are height and width of the beam.

The bending moment by putting Equation (20) into Equation (21) can be obtained as

$$
M_{x}=-D_{x x} \kappa_{x}-M_{T}
$$

The flexural rigidity of the beam and thermal moment are given by: $\begin{aligned} D_{x x} & =\int_{-\frac{h}{2}}^{\frac{h}{2}} b E(z, T) z^{2} d z \\ M_{T} & =\int_{-\frac{h}{2}}^{\frac{h}{2}} b E(z, T) T(z) \alpha(z, T) z d z\end{aligned}$

The elastic curve equation of the EBT beam can be expressed as [27]:

$$
\kappa_{x}=\frac{d^{2} w}{d x^{2}}=-\frac{M_{x}}{D_{x x}}
$$

Thus, Equation (24) is governing equation of classical beam which is used to analyze the clamped-clamped composite beam. Both sides clamped beam is statically indeterminate, therefore the superposition technique is obtained to puzzle out the governing equation of composite beam. This method as shown in Figure 11, the statically indeterminate beam is converted to several primary beams which is statically determinate and subjected to a separate reaction and loaded. The boundary conditions can be represented mathematically as:

$$
\begin{aligned}
& w_{1}+w_{2}+w_{3}+w_{4}=0 \quad \text { at } \quad x=a \\
& \frac{d w_{1}}{d x}+\frac{d w_{2}}{d x}+\frac{d w_{3}}{d x}+\frac{d w_{4}}{d x}=0 \quad \text { at } \quad x=a
\end{aligned}
$$

Where, $a$ is a length of the composite beam.
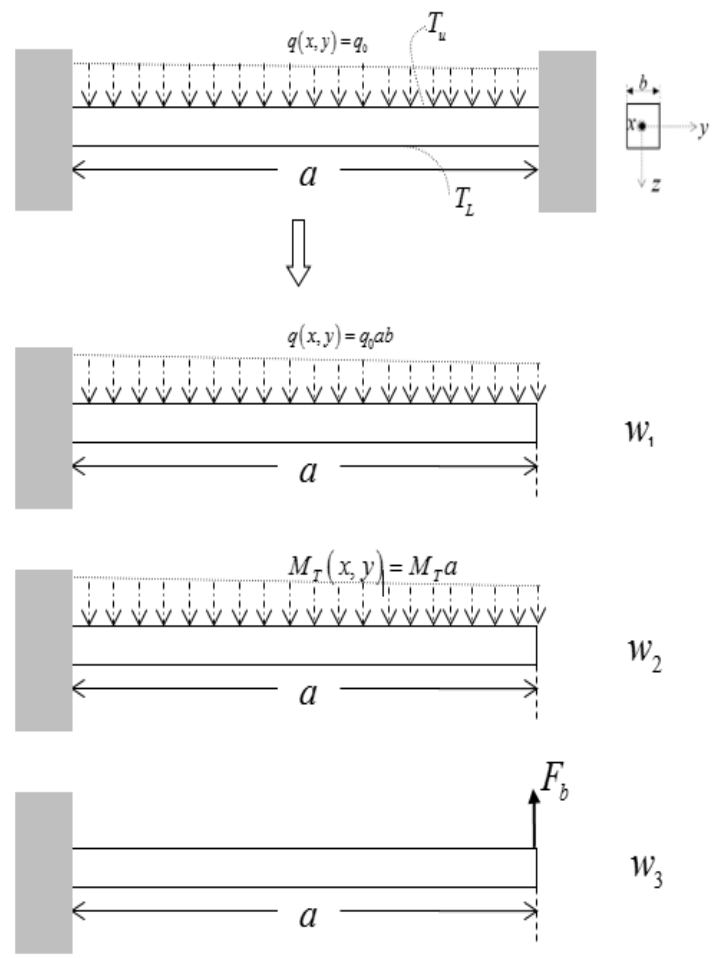

$w_{3}$

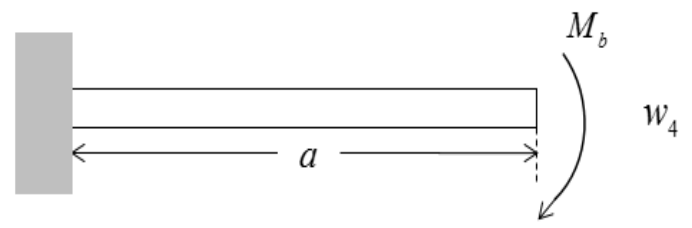

Fig. 11: Scheme superposition method of two sides clamped beam under distributed transverse load and exposed different temperature through the thickness of beam 


\subsection{Solution Composite Rectangular Clamped Beam}

As shown in Figure 11, by using superposition method, the deflection of the composite rectangular clamped beam is a linear combination of four deflections of cantilever beam problems.

$$
w=w_{1}+w_{2}+w_{3}+w_{4}
$$

The first beam problem is the cantilever beam subjected to uniformly distributed mechanical load (Figure 11). This problem can be determined by using the equation of the beam with boundary conditions.

$$
\begin{aligned}
& \frac{d^{2} w_{1}}{d x^{2}}=-\frac{M_{x 1}}{D_{x x}} \\
& w_{1}=0 \quad \text { and } \quad \frac{d w_{1}}{d x}=0 \quad \text { on } \quad x=0
\end{aligned}
$$

The bending moment at any section of the beam with $x$ distance from fixed edge is

$$
M_{x 1}=-\frac{q_{0} b x^{2}}{2}-\frac{q_{0} b a^{2}}{2}+q_{0} b a x
$$

Where, $q_{0}$ is a pressure load on the beam. Substituting Equation (28) into Eq. (27a) and then first and second integration of result equation with using boundary conditions (Equation 27b), gives slope and deflection equation:

$$
\begin{aligned}
& \frac{d w_{1}}{d x}=\frac{b q_{0}}{D_{x x}}\left[\frac{x^{3}}{6}+\frac{a^{2} x}{2}-\frac{a x^{2}}{2}\right] \\
& w_{1}=\frac{b q_{0}}{D_{x x}}\left[\frac{x^{4}}{24}+\frac{a^{2} x^{2}}{4}-\frac{a x^{3}}{6}\right]
\end{aligned}
$$

The second beam problem is the deflection $w_{2}$ at any point of the cantilever beam under uniformly distributed thermal moment $M_{T}$, which is exerted on the free end as shown in Figure 11. The governing equation and boundary conditions are

$$
\begin{aligned}
& \frac{d^{2} w_{2}}{d x^{2}}=-\frac{M_{x 2}}{D_{x x}} \\
& w_{2}=0 \quad \text { and } \quad \frac{d w_{2}}{d x}=0 \quad \text { on } \quad x=0
\end{aligned}
$$

The bending moment at any section of the beam with $x$ distance from fixed edge is

$$
M_{x 2}=-\frac{M_{T} x^{2}}{2}+M_{T} a x-\frac{M_{T} a^{2}}{2}
$$

Substituting Equation (32) into Equation (31a) and then first and second integration of result equation with using boundary conditions (Equation $31 \mathrm{~b}$ ), gives slope and deflection equation:

$$
\begin{gathered}
\frac{d w_{2}}{d x}=\frac{M_{T}}{D_{x x}}\left[\frac{x^{3}}{6}-\frac{a x^{2}}{2}+\frac{a^{2} x}{2}\right] \\
w_{2}=\frac{M_{T}}{D_{x x}}\left[\frac{x^{4}}{24}-\frac{a x^{3}}{6}+\frac{a^{2} x^{2}}{4}\right]
\end{gathered}
$$

The third beam problem is deflection $w_{3}$ at any point of the cantilever beam under single concentrated force $F_{b}$, which is exerted on the free end as shown in Figure 11. The governing equation and boundary conditions are

$$
\begin{aligned}
& \frac{d^{2} w_{3}}{d x^{2}}=-\frac{M_{x 3}}{D_{x x}} \\
& w_{3}=0 \quad \text { and } \quad \frac{d w_{3}}{d x}=0 \quad \text { on } \quad x=0
\end{aligned}
$$

The bending moment at any section of the beam with $x$ distance from fixed edge is

$$
M_{x 3}=F_{b} a-F_{b} x
$$

Substituting Equation (36) into Equation (35a) and then first and second integration of result equation with using boundary conditions (Equation 35b), gives slope and deflection equation:

$$
\begin{aligned}
& \frac{d w_{3}}{d x}=\frac{F_{b}}{D_{x x}}\left[\frac{x^{2}}{2}-a x\right] \\
& w_{3}=\frac{F_{b}}{D_{x x}}\left[\frac{x^{3}}{6}-\frac{a x^{2}}{2}\right]
\end{aligned}
$$

The fourth beam problem is the deflection $w_{4}$ at any point of the cantilever beam under the single moment $M_{b}$, which is exerted on the free end as shown in Figure 11-3. The governing equation and boundary conditions are

$$
\begin{aligned}
& \frac{d^{2} w_{4}}{d x^{2}}=-\frac{M_{x 4}}{D_{x x}} \\
& w_{4}=0 \quad \text { and } \quad \frac{d w_{4}}{d x}=0 \quad \text { on } \quad x=0
\end{aligned}
$$

The bending moment at any section of the beam with $x$ distance from fixed edge is

$$
M_{x 4}=-M_{b}
$$

Substituting Equation (40) into Equation (39a) and then first and second integration of result equation with using boundary conditions (Equation 39b), gives slope and deflection equation:

$$
\begin{aligned}
\frac{d w_{4}}{d x} & =\frac{M_{b}}{D_{x x}} x \\
w_{4} & =\frac{M_{b}}{2 D_{x x}} x^{2}
\end{aligned}
$$

The set of slope equations of four problems have to be compatible with clamped boundary condition. Thus, substituting Equations (29), (33), (37) and (41) into Equation (25b) at $x=a$ gives

$$
F_{b}=\frac{M_{T} a}{2}+\frac{q_{0} a}{2}
$$

The set of deflection equations of four problems have to be compatible with clamped boundary condition. Thus, substituting Equations (30), (34), (38) and (42) into Equation 25a, at $x=a$ gives

$$
M_{b}=\frac{2 a F_{a}}{3}-\frac{a^{2} M_{T}}{4}-\frac{q_{0} a^{2}}{4}
$$


By solving the system simultaneous equations (Equation (43) and Equation (44)), the unknown force and moment reactions at constrain $(x=a)$ are obtained:

$$
\begin{aligned}
& F_{b}=\frac{a b q_{0}}{2}+\frac{M_{T} a}{2} \\
& M_{b}=\frac{a^{2} b q_{0}}{12}+\frac{M_{T} a^{2}}{12}
\end{aligned}
$$

\section{Results and Discussion}

As noted initially, three models coated beam (HC, FGC, H-FGC) with the same thickness are considered for the purpose of analyzing two sides fixed rectangular coated beam, where HC and FGC models have a coated layer of $4 \mathrm{~mm}$ and metal substrate of $6 \mathrm{~mm}$, while the H-FGC model has three layers with a thickness ratio of 1:1:3. In all models of the composite the Poisson's ratio is 0.3.

The sample size of the three models are $50 \mathrm{~cm}$ by $5 \mathrm{~cm}$ by $1 \mathrm{~cm}$, above which a uniformly distributed mechanical load is applied at $q_{0}=22 \mathrm{kPa}$. The temperature on the top side and the bottom side are $T_{u}$ and $T_{L}=300^{\circ} \mathrm{K}$ respectively. For testing purposes, the models consider bending along the $\mathrm{z}$-axis as the primary mode of failure. In validating the results, a verification study is done for bending behavior due to uniformly transverse load and also for thermal loading. For mechanical loading verification, the equations of superposition method were validated with the isotropic homogeneous beam studies by R. C. Hibbeler [28] and for bending behaviour due to thermal load by Naotake Noda [29]. And also in order to verify the validity of the outputs obtained in the current paper, Table 4 is shown comparisons of the deflections at the center of $\mathrm{HC}$ beam under transverse uniformly distributed mechanical loads in Superposition Method (SM) with Energy Method (EM) and Finite Element Method (FEM). Table 4 shows wonderful concurrence, and therefore the SM solution of $\mathrm{HC}$ beam based on Euler-Bernoulli is valid.

Table 4: Comparisons of the deflection $(m)$ at the centre of clampedclamed HC beam under transverse uniformly distributed mechanical loading in the present paper to the deflections obtained by two other methods

\begin{tabular}{|c|c|c|c|}
\hline Ceramic / Metal & SM & EM & FEM \\
\hline$E_{h c}=10 \times 10^{10} / E_{h}=1 \times 10^{10}$ & $7.8639 \mathrm{e}-04$ & $7.8638 \mathrm{e}-04$ & $\begin{array}{c}7.8640 \mathrm{e}- \\
04\end{array}$ \\
\hline Silicon nitride / Stainless steel & $1.6241 \mathrm{e}-04$ & $1.6241 \mathrm{e}-04$ & $\begin{array}{c}1.6241 \mathrm{e}- \\
04\end{array}$ \\
\hline Zirconia / Ti - 6Al - 4V & $3.1449 \mathrm{e}-04$ & $3.1448 \mathrm{e}-04$ & $\begin{array}{c}3.1449 \mathrm{e}- \\
04\end{array}$ \\
\hline Aluminum oxide / Nickel & $1.6382 \mathrm{e}-04$ & $1.6382 \mathrm{e}-04$ & $\begin{array}{c}1.6382 \mathrm{e}- \\
04\end{array}$ \\
\hline$E_{h c}=$ Ceramic, $E_{h}=$ Metal & & & \\
\hline
\end{tabular}

Figures 12 and 14 show the deflections along the length of three different coated beam subjected to mechanical loading and thermo-mechanical loading respectively by considering different values of material parameters $(n)$. Since, the changes on the material properties of FGM depends on the Power-law function, the following graphs defines the FGM coated and under coated as P-FGC and H-P-FGC respectively. As shown in Figures 12 and 14 the deflection of beam in P-FGC model biger than other coated models, which with increasing amount of material parameters the deflections are decreased and became closer to the other coated models.

Figure 13 and 15 illustrate the variation of stress $\sigma_{x}$ cross the layers of beam at the center of the models under both mechanical and thermo-mechanical loading followed by stress figures (Figures 13, 15, 17 and 19) that show the problem of interface singularity between the ceramic coated layer and the substrate layer. But in P-FGC model and H-P-FGC model, a drastic reduction in stress singularity is observed, especially with increasing the material parameters in FGM layer. The stress figures show the magnitude of stresses on coated layer in P-FGC model compared to H-P-FGC model are significantly increased. As a resualt with increase the magnitude of stress on the coating layer in P-FGC model, the transverse displacement of the coated layer will be increased. Clearly, the superiority of H-P-FGC model coated against P-FGC model coated has been demonstrated.

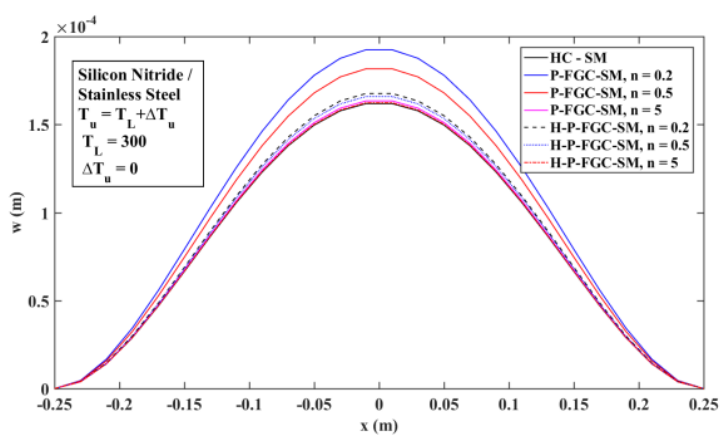

Fig. 12: Deflection the center of silicon nitride-stainless steel with three models coated beam under mechanical load

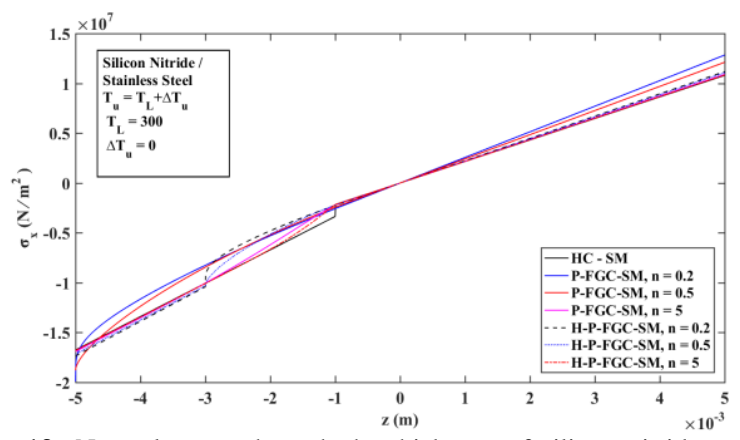

Fig. 13: Normal stress through the thickness of silicon nitride-stainless steel in three models at the centre of the beam under mechanical load

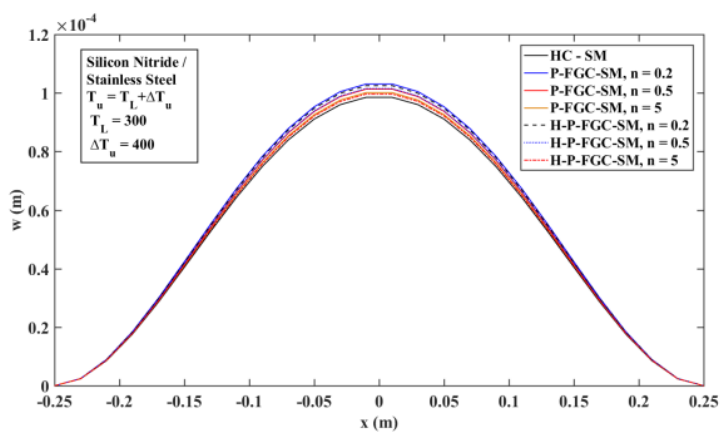

Fig. 14: Deflection the center of silicon nitride-stainless steel with three models coated beam under thermo-mechanical load

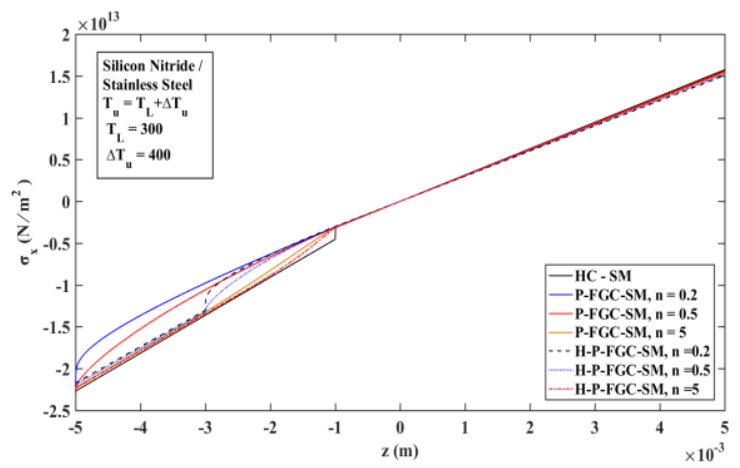

Fig. 15: Normal stress through the thickness of silicon nitride-stainless steel in three models at the centre of the beam under thermo-mechanical load 
Figure 16 and 18 show the deflections along the length of three different coated beam subjected to thermal loading and thermomechanical loading respectively by considering different values of material parameters $(n)$. The deflections of coated beams in absence of mechanical loading are upward in negative direction of coordinate.

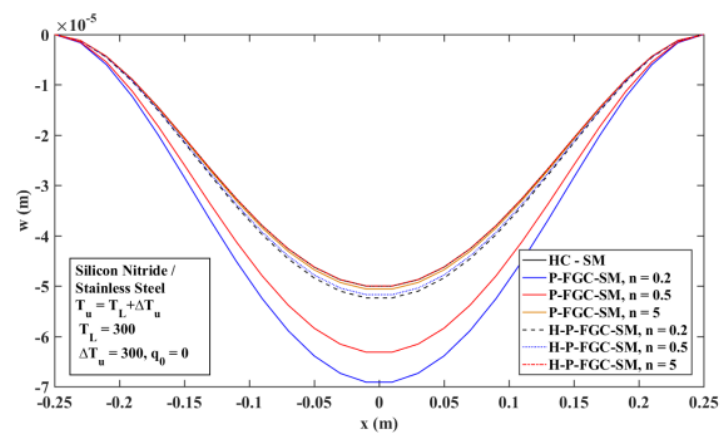

Fig. 16: Deflection the center of silicon nitride-stainless steel with three models coated beam under thermal load

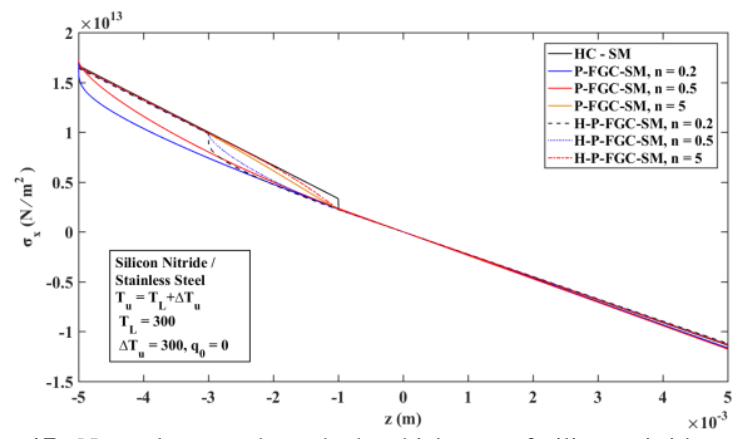

Fig. 17: Normal stress through the thickness of silicon nitride-stainless steel in three models at the centre of the beam under thermal load

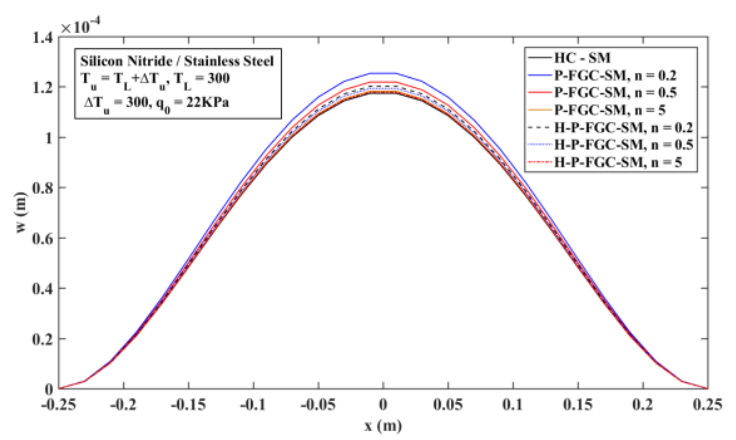

Fig. 18: Deflection the center of silicon nitride-stainless steel with three models coated beam under thermo-mechanical load

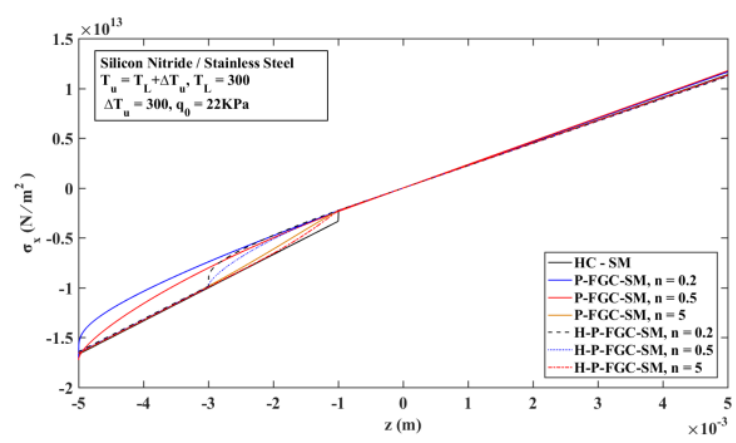

Fig. 19: Normal stress through the thickness of silicon nitride-stainless steel in three models at the centre of the beam under thermo-mechanical load

Figure 20 shows the deflection along the length of three different coated beam with three different couples material under thermo- mechanical loading with several material parameters. In case two the thermal expansion coefficient of ceramic (Zirconia) is much bigger than other ceramics. Therefore the deflections of coated beam in case two under similar loading is significantly different.

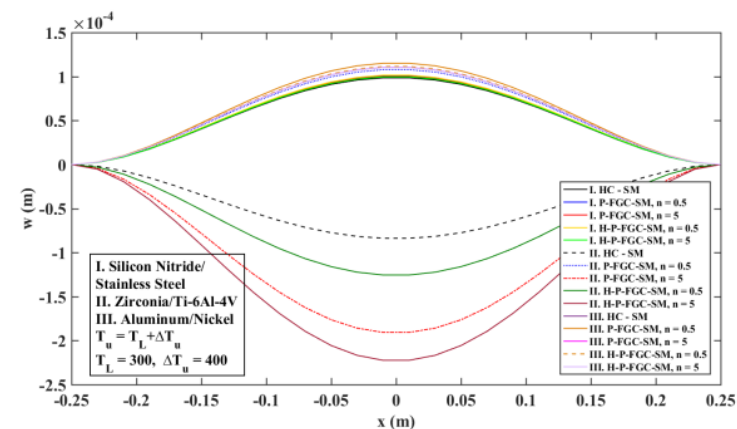

Fig. 20: Deflection the center of three models coated beam with three different couples' material under thermo-mechanical load

\section{Conclusion}

In this paper, the thermo-mechanical loading with variable temperature-dependent material properties is discussed in the context of a comparative study between a conventional coated beam and a FGM coated \& FGM under coated beam. The models consider the linearity in Euler-Bernoulli based on the power-law function applied. From the transverse displacement and the resultant stress along the beam with fully clamped boundary conditions by utilizing superposition method, there is a drastic reduction stress concentration at critical locations, demonstrating the superiority of FGM. As stated previously, in conventional coated beam, there is a stress concentration at interface of composite layers. But in FGM coated and FGM under coated beams, this stress concentration drastically decreased. In FGM coated model beam the transverse displacement is being much bigger than other models. In other words, the transverse displacement on the FGM under coated beam is very close to the conventional coated beam. As a result, in the FGM under coated beam, not only is the stress concentration problem less than the conventional coated beam, but also the transverse displacement in the FGM under coated beam under thermal and mechanical loading is almost like the conventional coated beam. Clearly, the superiority of FGM under coated composite against conventional coated and FGM coated composite has been demonstrated.

The other result is that the material properties of the couple materials which is used in composite have vital role on results. An example application of FGM is between Zirconia, Aluminum oxide and Silicon nitride. The difference in coefficient of thermal expansion generates transverse displacement much differ to other pairs of materials. Therefore, the transverse displacement of Zirconia / Ti-6Al-4V is much differ compared with other pairs of materials.

\section{Acknowledgement}

The support of this research by Universiti Tenaga Nasional (Uniten) under higher education of federal government of Malaysia through Grant 06012012ERGS, is gratefully acknowledged.

\section{References}

[1] S. Suresh and A. Mortensen, Fundamentals of functionally graded materials: The Institut of Materials, 1998.

[2] M. Koizumi, "FGM activities in Japan," Composites Part B: Engineering, vol. 28, pp. 1-4, 1997.

[3] M. Koizumi and M. Niino, "Overview of FGM research in Japan," Mrs Bulletin, vol. 20, pp. 19-21, 1995. 
[4] W. A. Kaysser and B. Ilschner, "FGM research activities in Europe," Mrs Bulletin, vol. 20, pp. 22-26, 1995.

[5] Q. Xia and M. Y. Wang, "Simultaneous optimization of the material properties and the topology of functionally graded structures," Computer-Aided Design, vol. 40, pp. 660-675, 2008.

[6] Z. Jingchuan, Y. Zhongda, L. Zhonghong, and L. Jian, "Microstructure and thermal relaxation of $\mathrm{ZrO} 2 / \mathrm{Ni}$ functionally graded materials," Trans. Nonferrous Met. Soc. China, vol. 6, pp. 94-99, 1996.

[7] K. Tohgo, M. Iizuka, H. Araki, and Y. Shimamura, "Influence of microstructure on fracture toughness distribution in ceramic-metal functionally graded materials," Engineering Fracture Mechanics, vol. 75, pp. 4529-4541, 2008.

[8] M. Bocciarelli, G. Bolzon, and G. Maier, "A constitutive model of metal-ceramic functionally graded material behavior: formulation and parameter identification," Computational Materials Science, vol. 43, pp. 16-26, 2008.

[9] X. Jin, L. Wu, L. Guo, H. Yu, and Y. Sun, "Experimental investigation of the mixed-mode crack propagation in $\mathrm{ZrO} 2 / \mathrm{NiCr}$ functionally graded materials," Engineering Fracture Mechanics, vol. 76, pp. 1800-1810, 2009.

[10] M. El-Wazery and A. El-Desouky, "A review on functionally graded ceramic-metal materials," Journal of Materials and Environmental Science, vol. 6, pp. 1369-1376, 2015.

[11] N. Wattanasakulpong, "Thermal buckling and elastic vibration analysis of functionally graded beams and plates using improved third order shear deformation theory," School of Mechanical and Manufacturing Engineering, The University of New South Wales, 2012.

[12] Y. Touloukian, R. Powell, C. Ho, and M. Nicolaou, "Thermophysical Properties of Matter-The TPRC Data Series. Volume 10. Thermal Diffusivity," Thermophysical and Electronic Properties Information Analysis Center Lafayette In 1974.

[13] H.-S. Shen, "Nonlinear bending response of functionally graded plates subjected to transverse loads and in thermal environments," International Journal of Mechanical Sciences, vol. 44, pp. 561-584, 2002.

[14] J. Reddy and C. Chin, "Thermomechanical analysis of functionally graded cylinders and plates," Journal of thermal Stresses, vol. 21, pp. 593-626, 1998

[15] Z.-Q. Cheng and R. Batra, "Exact correspondence between eigenvalues of membranes and functionally graded simply supported polygonal plates," Journal of Sound and Vibration, vol. 229, pp. 879-895, 2000.

[16] H. Navazi and H. Haddadpour, "Aero-thermoelastic stability of functionally graded plates," Composite Structures, vol. 80, pp. 580587,2007

[17] S. Sina, H. Navazi, and H. Haddadpour, "An analytical method for free vibration analysis of functionally graded beams," Materials \& Design, vol. 30, pp. 741-747, 2009.

[18] M. Ganapathi, "Dynamic stability characteristics of functionally graded materials shallow spherical shells," Composite structures, vol. 79, pp. 338-343, 2007.

[19] D. Sun and S.-N. Luo, "Wave propagation and transient response of functionally graded material circular plates under a point impact load," Composites Part B: Engineering, vol. 42, pp. 657-665, 2011.

[20] H.-S. Shen, Functionally graded materials: nonlinear analysis of plates and shells: CRC press, 2016.

[21] Y.-L. Chung and W.-T. Chen, "Bending behavior of FGM-coated and FGM-undercoated plates with two simply supported opposite edges and two free edges," Composite Structures, vol. 81, pp. 157167, 2007.

[22] S. Caraballo, "Thermo-Mechanical Beam Element for Analyzing Stresses in Functionally Graded Materials," 2011.

[23] M. Zaki, F. Tarlochan, and S. Ramesh, "Two dimensional elastic deformations of functionally graded coated plates with clamped edges," Composites Part B: Engineering, vol. 45, pp. 1010-1022, 2013

[24] R. Javaheri and M. Eslami, "Thermal buckling of functionally graded plates based on higher order theory," Journal of thermal stresses, vol. 25, pp. 603-625, 2002.

[25] E. Carrera, G. Giunta, and M. Petrolo, Beam structures: classical and advanced theories: John Wiley \& Sons, 2011.

[26] C. Wang, J. N. Reddy, and K. Lee, Shear deformable beams and plates: Relationships with classical solutions: Elsevier, 2000

[27] R. C. Hibbeler, Mechanics of Materials MasteringEngineering Access Code: Includes Pearson EText: Pearson College Division, 2013.
[28] R. C. Hibbeler, Mechanics of Materials Plus Masteringengineering with Pearson Etext -- Access Card Package: Pearson Education, 2016.

[29] N. Noda, Thermal stresses: CRC press, 2002. 